\title{
Development of a laboratory-based X-ray absorption system for energy material research
}

\author{
Ruimin Qiao ${ }^{1 *}$, Srivatsan Seshadri ${ }^{*}$, Sylvia Lewis ${ }^{1}, \mathrm{SH} \mathrm{Lau}^{1}$ and Wenbing Yun ${ }^{1}$ \\ 1. Sigray, Inc., 5750 Imhoff Drive, Suite I, Concord, CA, USA. \\ * Corresponding author: rqiao@sigray.com, srivatsan.seshadri@sigray.com
}

It is becoming increasing clear that clean energy is imperative for our sustainable future and requires development of inexpensive large-scale energy storage systems for wide deployment. Li-ion batteries, based on $\mathrm{LiCoO}_{2}$ as cathode materials for example, are already widely used in consumer electronics and wireless communication segments and further upscaling of storage systems require cheaper cathode materials and Lithium Iron Phosphate $\left(\mathrm{LiFePO}_{4}\right)$ is one of the promising candidates but suffer from low intrinsic electrical conductivity [1]. Redox flow batteries, Vanadium redox battery for example, also offer promise of highly efficient systems but suffer from the need for expensive membranes and limitations of active material concentration and high cost. Li-redox flow batteries, such as Fe-based Liredox batteries, seek to exploit the advantages of Li-ion and redox-flow batteries [2].

X-Ray Absorption Spectroscopy (XAS) is a technique that is particularly suited to study battery materials because of its ability to study a particular element of interest by probing its $\mathrm{K}$ (or L) absorption edge and extract information regarding the chemical states such as oxidation state by XANES (x-ray absorption near edge structure), interatomic distances and coordination number by EXAFS (extended xray absorption fine structure) in materials.

XAS is predominantly performed at synchrotron facilities due to their high brightness and tunable energy x-ray beams $[3,4]$. However, difficulty in timely access, limited beamtime and challenging logistic issues limits the scope of their use and preclude the possibility of using them for routine measurements. Hence, there is significant need for a high throughput and high performance laboratorybased XAS system that enables academic and industrial researchers perform routine measurements and analyses on various samples.

We have developed a compact, laboratory based, vacuum compatible XAS system operating in the 2$12 \mathrm{KeV}$ energy range with major capabilities such as sub eV resolution over a fairly large energy range. and short data acquisition times (seconds to minutes). This performance is achieved by using internally developed x-ray source, highly efficient double paraboloid x-ray optics with a sharp high energy cut off (which allows for reduction of higher order harmonics), single crystal x-ray crystal analysers based on Johansson geometry (for XANES which require high energy resolution) and HOPG crystals for EXAFS which can tolerate a more relaxed energy resolution $(\sim 10 \mathrm{eV})$, and a high resolution spatially resolving $2 \mathrm{D}$ detector.

As a demonstration of the performance of this laboratory-based system, the XANES spectra of $\mathrm{V}$ and Fe K-edge collected are displayed and compared with synchrotron data in figure 1 . The data collected using laboratory-based system is consistent with synchrotron data and has similar energy resolution. In this presentation, we will review the system design, innovations and describe experimental results in greater detail [5]. 


\section{References:}

[1] Ting Zhao, Wei Xu, Qing Ye, Jie Cheng, Haifeng Zhao, Ziyu Wu, Dingguo Xiac and Wangsheng Chua, J. Synchrotron Rad., 17 (2010) p. 584-589

[2] Yu Zhao,Yu Ding, Yutao Li, Lele Peng, Hye Ryung Byon, John B. Goodenough and Guihua Yu, Chem. Soc. Rev., 44 (2015) p. 7968-7996.

[3] Won-Sub Yoon, Clare P. Grey, Mahalingam Balasubramanian, Xiao-Qing Yang and James McBreen, Chemistry of Materials 15 (2003) p. 3161-3169.

[4] Janis Timoshenko, Deyu Lu, Yuewei Lin, and Anatoly I. Frenkel, The Journal of Physical Chemistry Letters 8 (2017) p. 5091-5098.

[5] The authors gratefully acknowledge funding from the NIH, National Cancer Institute for the development of the XANES system (R44CA228912).
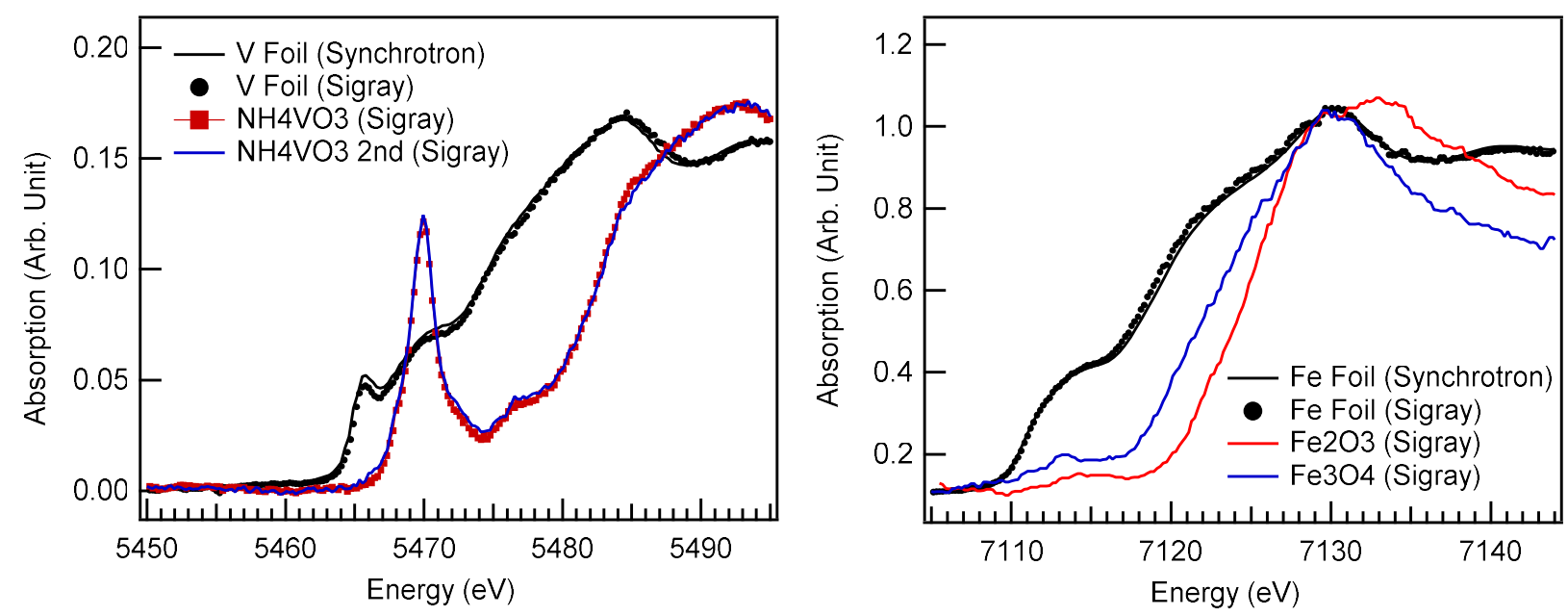

Figure 1. (A) XANES spectra of $\mathrm{V}$ in $\mathrm{V}$ foil and $\mathrm{NH}_{4} \mathrm{VO}_{3}$. (B) XANES spectra of $\mathrm{Fe}$ in $\mathrm{Fe}$ foil, $\mathrm{Fe}_{2} \mathrm{O}_{3}$ and $\mathrm{Fe}_{3} \mathrm{O}_{4}$. The data collected using laboratory-based system is consistent with synchrotron data and has similar energy resolution. As indicated by the dramatically different spectrum line shapes, XANES is a powerful technique for chemical state analysis. 